\title{
Lamivudine Therapy Exacerbates Bilirubinemia in Patients Underlying Severely Advanced Hepatitis
}

\author{
Young Hee Choi', Chang Ho Lee ${ }^{2}$, Myong Suk Ko ${ }^{3}$, Hyun Joo Han ${ }^{4}$ and Sang Geon Kim ${ }^{4,5}$ \\ ${ }^{1}$ College of Pharmacy, Dongguk University_Seoul, Seoul, Korea \\ ${ }^{2}$ Department of Pharmacology, College of Medicine, Hanyang University, Seoul, Korea \\ ${ }^{3}$ Korea Intellectual Property Strategy Agency, Business Cooperation Team, Seoul, Korea \\ ${ }^{4}$ Department of Pharmacy, Seoul National University Hospital, Seoul, Korea \\ ${ }^{5}$ College of Pharmacy and Research Institute of Pharmaceutical Sciences, Seoul National University, Seoul, Korea
}

\begin{abstract}
Lamivudine belongs to the set of antiviral agents effective against hepatitis B virus infection. Given case reports on liver injuries after certain antiviral agent treatments, this study examined the effects of lamivudine on alanine aminotransferase (ALT) and total bilirubin (TB) using a medical system database. A total of 1,321 patients taking lamivudine alone or with others were evaluated using laboratory hits in an electronic medical system at Seoul National University Hospital from 2005 through 2011. The patients were grouped according to prior ALT results: G\#1, ALT < 40 IU/L; G\#2, 40 IU/L $\leq$ ALT $<120$ IU/L; G\#3, 120 $\mathrm{IU} / \mathrm{L} \leq \mathrm{ALT}<240 \mathrm{IU} / \mathrm{L}$; and G\#4, ALT $\geq 240 \mathrm{IU} / \mathrm{L}$. In G\#1 and G\#2 patients, lamivudine or adefovir treatment decreased ALT and TB compared to prior values. In G\#3 and G\#4 patients with three times the upper limit of normal $(\mathrm{ULN}) \leq$ ALT $<15$ times the ULN, both ALT and TB were decreased after treatment with lamivudine alone, or adefovir following lamivudine therapy, indicating that lamivudine therapy ameliorated liver functions. However, in G\#4 patients who experienced severely advanced hepatitis (ALT $\geq 15$ times the ULN, or $\geq 600 \mathrm{IU} / \mathrm{L})$, lamivudine augmented TBmax $(6.3 \rightarrow 13.3 \mathrm{mg} / \mathrm{dL})$ despite a slight improvement in ALT $(839 \rightarrow 783 \mathrm{IU} / \mathrm{L})$, indicative of exacerbation of bilirubinemia. Patients who used adefovir after lamivudine also showed a high incidence of hyperbilirubinemia when they experienced severely advanced hepatitis. Treatment with adefovir alone did not show the effect. In conclusion, lamivudine may increase the risk of hyperbilirubinemia in patients with severely advanced hepatitis, implying that caution should be exercised when using lamivudine therapy in certain patient populations.
\end{abstract}

Key words: Lamivudine, Drug-associated hyperbilirubinemia, Laboratory signal hits, Total bilirubin, ALT

\section{INTRODUCTION}

Chronic hepatitis B virus (HBV)-infected patients are characterized by high alanine aminotransferase (ALT) activities, possibly accompanying jaundice and hepatic decompensation (1). Lamivudine, a cytosine nucleoside analogue, inhibits viral replication by acting on HBV DNA polymerase

Correspondence to: Sang Geon Kim, Department of Pharmacy, Seoul National University Hospital; College of Pharmacy and Research Institute of Pharmaceutical Sciences, Seoul National University, Seoul 08826, Korea

E-mail: sgk@snu.ac.kr

Abbreviations: ADR, adverse drug reaction; ALT, alanine aminotransferase; AST, aspartate transaminase; EMR, electronic medical record; TB, total bilirubin (TB); UDCA, ursodeoxycholic acid; ULN, upper limit of normal. and inducing chain termination. Lamivudine improves the parameters of liver function and histopathology, such as fibrosis, HBeAg seroconversion, or ALT normalization (2-7). Generally, lamivudine therapy allows HBV-infected patients to recover ALT and/or aspartate transaminase (AST) activities to normal ranges through virus eradication and is often recommended for long-term treatment rather than limited 
course treatment (8). It is also administered with other drugs, such as adefovir and entecavir, to enhance antiviral efficacy.

It should be noted, however, that some anti-viral agents, particularly those requiring a high-dosage treatment regimen, have the potential to initiate chemical-induced liver injury. Due to the burden of metabolic detoxification, especially for those with underlying severely diminished liver function, caution should be exercised when using antiviral therapy. In fact, the possibility of adverse drug reaction (ADR) occurrences induced by lamivudine and/or other anti-viral agents has been proposed in some case studies, e.g., lamivudine treatment acutely exacerbated hepatocyte injury in those with chronic hepatitis (8). Therefore, lamivudine-induced liver injury particularly in patients suffering from advanced liver diseases is possible. Moreover, large-scale database analyses for this possibility have not been carried out. Thus, an understanding of the ADRs of anti-viral agents in the context of liver dysfunction may be of assistance to accomplish successful long-term therapy.

To create a data-acquisition method using electronic medical records (EMRs) and apply the result for the assessment of ADR types and incidences, factors such as patient laboratory signals, genders, ages, organ functions, pathological factors, and co-administered drugs should be analyzed (9). In addition, drug-associated factors may be deduced from incidence rates, severity, duration time, and onset time of the parameters of interest. For liver function, cholestasis, and other liver-related disease conditions, parameters including total bilirubin (TB) and ALT and AST activities in blood may provide clues $(10,11)$. In general, ALT and AST activity indicate the severity of hepatitis, whereas blood bilirubin content reflects the extent of not only hepatocyte injury, but detoxifying capacity. Hence, blood bilirubin content generally reflects liver function as bilirubin is produced when the liver removes heme derived from old red blood cells. In most cases, hyperbilirubinemia causes jaundice, which may be further exacerbated by pathologic hepatic conditions and hepatitis.

It has been claimed that anti-viral treatment regimens containing a fixed-dose combination of paritaprevir, ritonavir, and ombitasvir may cause serious liver injury in patients with underlying advanced liver disease (12), suggesting that certain antiviral medications impose metabolic burdens to patients presumably due to the drug intermediates produced. Also, relatively larger doses of lamivudine are given compared to other anti-viral agents, with common oral doses of lamivudine, adefovir, and entecavir being 300, 10, and 0.5 $\mathrm{mg} /$ day, respectively (13-15). Given these points, this study assessed the effects of lamivudine treatment alone or in conjunction with adefovir using parameters representing liver function in groups of patients with mild, moderate, or severe hepatitis. We found that lamivudine alone or adefovir after lamivudine therapy may enhance the risk of hyperbilirubinemia in patients with severely advanced hepatitis.

\section{MATERIALS AND METHODS}

Study population. This study was approved by the Institutional Review Board of Seoul National University Hospital (SNUH, IRB No. 1012-046-344), which is a 1,961-bed medical center in Korea. The database contained records of 496,530 patients ( $\geq 18$ years), who had been admitted to SNUH and had undergone liver function tests from January 2005 through December 2011. The retrieved data had anonymous codes representing patient files comprising age, gender, medical diagnosis codes, blood ALT and AST activities, TB contents, dates of laboratory samples drawn, and medications (including generic or brand name, prescription date, and duration).

Patients. Patients were 18 years or older, were prescribed with lamivudine, adefovir, and/or entecavir, and had at least three data points of ALT, AST, albumin, and TB measurements during anti-viral therapy documented in their medical records. As a result, laboratory data were obtained from 353 patients using the EMR system. They were divided into four groups according to prior ALT ranges; G\#1, ALT $<40 \mathrm{IU} / \mathrm{L} ; \mathrm{G \# 2}, 40 \mathrm{IU} / \mathrm{L} \leq \mathrm{ALT}<120 \mathrm{IU} / \mathrm{L} ; \mathrm{G \# 3}, 120 \mathrm{IU} / \mathrm{L}$ $\leq \mathrm{ALT}<240 \mathrm{IU} / \mathrm{L}$; and $\mathrm{G} \# 4, \mathrm{ALT} \geq 240 \mathrm{IU} / \mathrm{L}$. In each group, other parameters were also retrieved to monitor changes in liver function prior to or during lamivudine treatment. Maximum total bilirubin (TBmax) content was used to assess drug effects on liver function because the content represents the functional remaining liver fraction (i.e., the detoxifying capacity).

Data acquisition and the mining process. TB measurements were sorted into categories according to the preexisting history of use of lamivudine, adefovir, entecavir, and other concomitant drug treatments. The steps for extraction and the data mining process are presented in Supplementary Fig. 1. Inclusion or exclusion criteria for each laboratory signal (i.e., ALT and TB in the blood) were critical for optimal patient grouping. First, patients having one or two data points for either ALT and TB were excluded. Second, TB contents determined prior to the first measurement were assumed to be in a normal range of $<1.4 \mathrm{mg} / \mathrm{dL}$. Similarly, ALT and AST activities were considered within a normal range when $<40 \mathrm{mg} / \mathrm{dL}$. They were used as cut-offs for bilirubinemia or abnormal liver function. Third, the incidence rate of hyperbilirubinemia was expressed as a percentage in each group. Data is shown as the median (minimum-maximum) in Tables 1 and 2. To assess the potential of drug-induced hyperbilirubinemia, patients who used adefovir after lamivudine therapy were additionally analyzed. 
Data analysis. Microsoft Excel and Access software and Microsoft Excel functions (version 2013) were used to analyze ALT, TB, and the other parameters. The results were considered statistically significant if the $p$-value was less than 0.05 . Statistical analyses were conducted using SPSS version 12.0 (SPSS Inc., Chicago, IL, USA).

\section{RESULTS}

\section{Analysis of patients subjected to lamivudine therapy.}

The baseline characteristics of patients is summarized in Table 1. In those who had no hepatitis (G\#1), either serum ALT activities or TB contents were not significantly different during the period of lamivudine therapy as compared to the baselines. In G\#1, lamivudine treatment had no effect on our parameters of interest. In G\#2, lamivudine decreased ALT by $35 \%$; TBmax values were reduced to a greater degree (72\%; 2.5 to $0.9 \mathrm{mg} / \mathrm{dL}$ ) (Fig. 1A). In G\#3, ALT activity and TBmax were decreased $24.7 \%$ and $45.9 \%$, respectively. Thus, lamivudine therapy lowered TBmax to a larger extent than ALT in patients who had a moderate degree of hepatitis. In G\#2 and G\#3, ALT and TB were widely scattered after lamivudine treatment compared to the baselines observed prior to therapy. In $\mathrm{G} \# 4$, lamivudine treatment ameliorated TBmax less than ALT (3.7\% versus
9.8\%) (Fig. 1B). Intriguingly, administration of lamivudine to a subgroup of patients who had experienced severely advanced hepatitis (i.e., ALT $\geq 15$ times ULN; ALT $\geq 600$ $\mathrm{IU} / \mathrm{L}$ ) greatly increased TBmax (from 6.3 to $13.3 \mathrm{mg} / \mathrm{dL}$ ), although ALT was slightly improved (from 839 to $783 \mathrm{IU} / \mathrm{L}$ ).

Next, we performed sub-group analyses for patients in $\mathrm{G} \# 3$ and $\mathrm{G} \# 4$ using a TBmax cut-off of $4.2 \mathrm{mg} / \mathrm{dL}$ (i.e., three times ULN). ALT and TB, and the differences between prior to use and during use of lamivudine medication, are summarized in Table 2. $\mathrm{ALT}_{0}$ and ALTmax were $77.9 \%$ and $81.4 \%$ greater, respectively, in patients with high TBmax (i.e., $\geq 4.2 \mathrm{mg} / \mathrm{dL}$ ) than those with low TBmax (i.e., $<4.2 \mathrm{mg}$ / dL). In high TBmax patients, neither ALT nor TB were improved. By contrast, low TBmax patients showed an improvement in TB, but not in ALT, suggesting that TB content may represent functional liver fraction more sensitively.

Another correlation analysis was done on ALT differences and TBmax. Patients showing abnormal TBmax were $19.3 \%(\mathrm{n}=32$ patients having abnormal TBmax versus a total of 166 patients), $13.3 \%$ (11 versus 83 ), 31.1\% (14 versus 45 ) and $79.7 \%$ (47 versus 59 ) in $\mathrm{G} \# 1, \mathrm{G} \# 2, \mathrm{G} \# 3$, and $\mathrm{G} \# 4$, respectively. The data showing abnormal TBmax were re-analyzed for ALT differences and TBmax. Among the patients with abnormal TBmax in $\mathrm{G} \# 1$ and $\mathrm{G} \# 2$, there was a positive correlation between ALT increases and TBmax

Table 1. ALT activities and TB contents before and during antiviral therapy

\begin{tabular}{|c|c|c|c|c|}
\hline & G1 & G2 & G3 & G4 \\
\hline Lamivudine & $\mathrm{n}=166$ & $\mathrm{n}=83$ & $\mathrm{n}=45$ & $\mathrm{n}=59$ \\
\hline Age (years) & $53(21 \sim 63)$ & $48(24 \sim 61)$ & $59(21 \sim 58)$ & $55(25 \sim 69)$ \\
\hline Gender $(\mathrm{M}, \mathrm{F})$ & 89,77 & 47,36 & 30,15 & 32,27 \\
\hline \multicolumn{5}{|l|}{ ALT (IU/L) } \\
\hline Before & $25(12 \sim 38)$ & $89(42 \sim 118)$ & $192(121 \sim 216)$ & $592(253 \sim 1348)$ \\
\hline During & $38(8.1 \sim 43)$ & $63(21 \sim 125)$ & $183(64 \sim 954)$ & $455(91 \sim 1065)$ \\
\hline \multicolumn{5}{|l|}{$\mathrm{TB}(\mathrm{mg} / \mathrm{dL})$} \\
\hline Before & $1.8(0.2 \sim 3.2)$ & $2.5(1.2 \sim 4.3)$ & $6.1(4.3 \sim 7.6)$ & $8.1(4.8 \sim 9.6)$ \\
\hline During & $0.8(0.3 \sim 3.5)$ & $0.9(0.8 \sim 4.3)$ & $3.3(0.4 \sim 18.3)$ & $6.8(0.8 \sim 11.3)$ \\
\hline Adefovir & $\mathrm{n}=103$ & $n=63$ & $\mathrm{n}=41$ & $\mathrm{n}=42$ \\
\hline Age (years) & $51(23 \sim 65)$ & $47(22 \sim 65)$ & $53(20 \sim 59)$ & $51(22 \sim 71)$ \\
\hline Gender $(\mathrm{M}, \mathrm{F})$ & 51,52 & 31,32 & 15,26 & 12,30 \\
\hline \multicolumn{5}{|l|}{ ALT (IU/L) } \\
\hline Before & $21(11 \sim 36)$ & $72(43 \sim 102)$ & $171(121 \sim 222)$ & $512(242 \sim 912)$ \\
\hline During & $29(10 \sim 43)$ & $63(32 \sim 115)$ & $152(95 \sim 624)$ & 355 (98 998) \\
\hline \multicolumn{5}{|l|}{$\mathrm{TB}(\mathrm{mg} / \mathrm{dL})$} \\
\hline Before & $1.8(0.2 \sim 3.2)$ & $2.2(1.2 \sim 4.3)$ & $5.1(4.3 \sim 7.6)$ & $7.1(4.8 \sim 8.6)$ \\
\hline During & $1.6(0.3 \sim 3.8)$ & $1.3(0.8 \sim 3.9)$ & $4.5(0.6 \sim 8.2)$ & $7.5(1.2 \sim 10.3)$ \\
\hline Entacavir & $\mathrm{n}=85$ & $\mathrm{n}=63$ & $\mathrm{n}=32$ & $\mathrm{n}=38$ \\
\hline Age (years) & $50(22 \sim 60)$ & $49(26 \sim 58)$ & $52(23 \sim 68)$ & $48(21 \sim 63)$ \\
\hline Gender $(\mathrm{M}, \mathrm{F})$ & 39,46 & 37,26 & 11,21 & 20,18 \\
\hline \multicolumn{5}{|l|}{ ALT (IU/L) } \\
\hline Before & $17(11 \sim 38)$ & $71(41 \sim 117)$ & $155(121 \sim 201)$ & 465 (242 819) \\
\hline During & $30(13 \sim 43)$ & $63(26 \sim 110)$ & $147(69 \sim 305)$ & $316(153 \sim 965)$ \\
\hline \multicolumn{5}{|l|}{$\mathrm{TB}(\mathrm{mg} / \mathrm{dL})$} \\
\hline Before & $1.4(0.2 \sim 2.3)$ & $2.2(1.3 \sim 4.4)$ & $5.1(2.3 \sim 7.6)$ & $5.6(2.6 \sim 8.6)$ \\
\hline During & $1.3(0.3 \sim 3.8)$ & $1.3(0.6 \sim 4.3)$ & $3.5(0.6 \sim 8.2)$ & $3.9(1.1 \sim 10.3)$ \\
\hline
\end{tabular}


(A) G\#1 and G\#2 patients with lamivudine

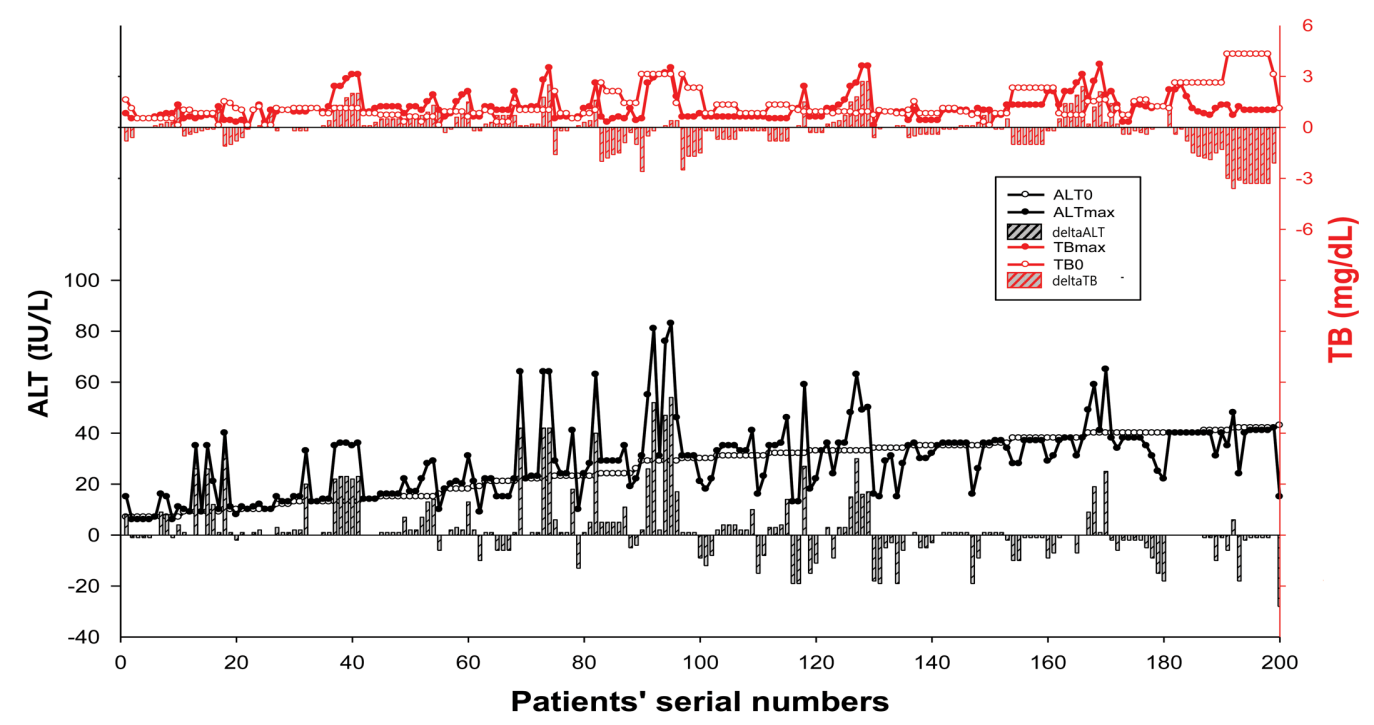

(B) G\#3 and G\#4 patients with lamivudine
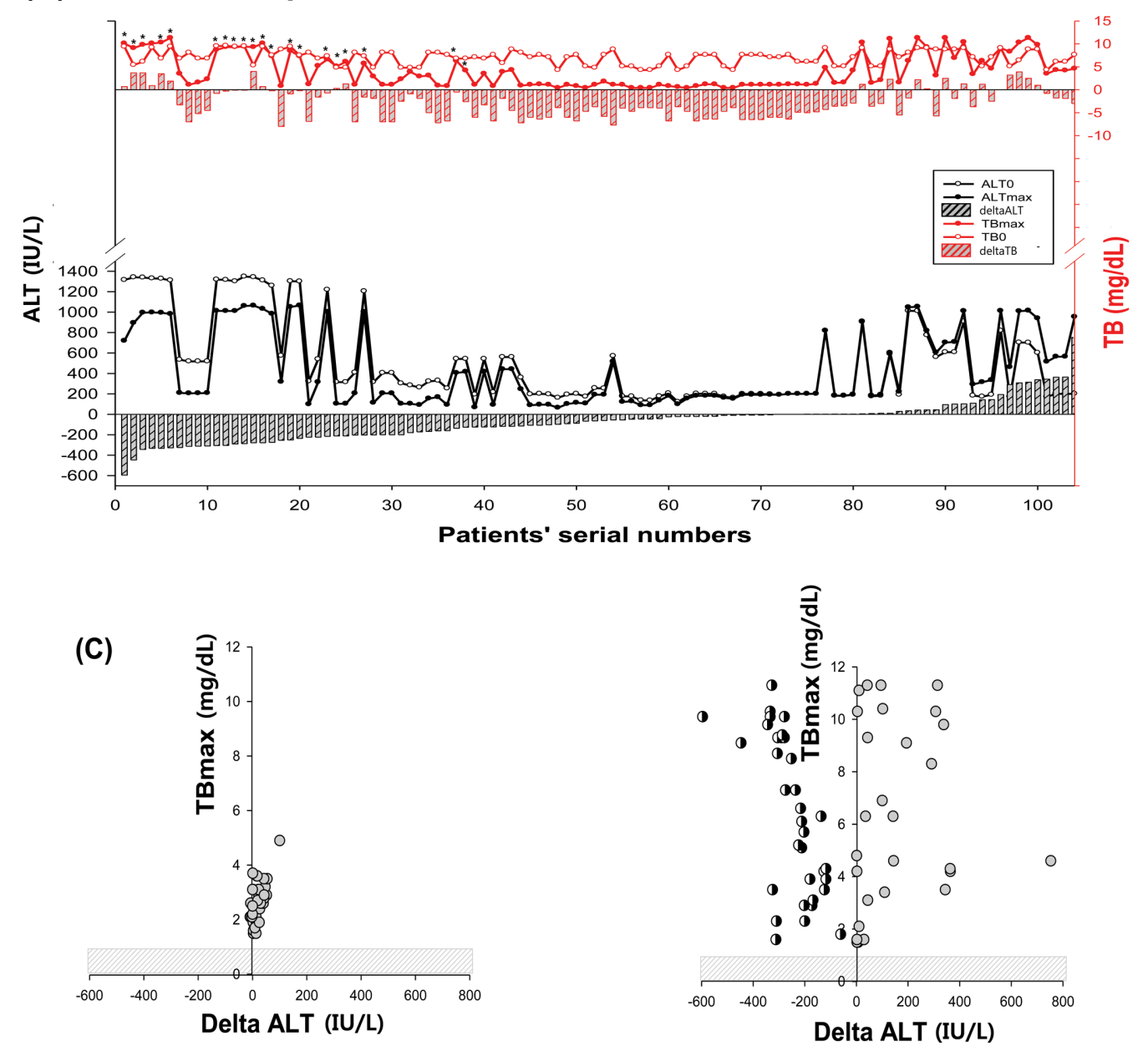

Fig. 1. (A, B) $A L T_{0}, A L T_{\max }, T B_{0}$, and $T_{\max }$ values before and during lamivudine therapy in $G \# 1$ and $G \# 2$ groups or in $G \# 3$ and $G \# 4$ groups. The differences between prior to and during therapy are shown as delta ALT (IU/L) or delta TB (mg/dL). (C) The relationship between delta ALT (IU/L) and $\mathrm{TB}_{\max }\left(\mathrm{mg} / \mathrm{dL}\right.$ ) in patients showing $\mathrm{TB}_{\max }>1.4 \mathrm{mg} / \mathrm{dL}$ in $\mathrm{G \# 1}$ and G\#2 groups (left) or in G\#3 and G\#4 groups (right; 1 , patient with an increase of ALT; and $\mathbf{O}$, patient with a decrease of ALT). 
Table 2. ALT activities and TB contents before and during antiviral therapy in G\#3 and G\#4

\begin{tabular}{|c|c|c|c|c|}
\hline & \multicolumn{2}{|c|}{$3 \times \mathrm{ULN} \leq \mathrm{ALT}<15 \times \mathrm{ULN}$} & \multicolumn{2}{|c|}{$\mathrm{ALT} \geq 15 \times \mathrm{ULN}$} \\
\hline & Delta ALT (-) & Delta ALT $(+)$ & Delta ALT (-) & Delta ALT $(+)$ \\
\hline \multicolumn{5}{|c|}{ Lamivudine $(\mathrm{n}=103)$} \\
\hline Delta TB (-) & $62^{*}$ & 3 & 2 & 1 \\
\hline Delta TB $(+)$ & $6^{*}$ & 12 & $13^{*}$ & 4 \\
\hline \multicolumn{5}{|l|}{ Adefovir $(\mathrm{n}=83)$} \\
\hline Delta TB (-) & $39^{*}$ & 10 & 4 & 0 \\
\hline Delta TB $(+)$ & 8 & 11 & $10^{*}$ & 1 \\
\hline \multicolumn{5}{|c|}{ Adefovir alone $(n=53)$} \\
\hline Delta TB (-) & $27^{*}$ & 6 & 3 & 0 \\
\hline Delta TB $(+)$ & 4 & 9 & 3 & 1 \\
\hline \multicolumn{5}{|c|}{ Lamivudine + Adefovir $(n=30)$} \\
\hline Delta TB (-) & $12^{*}$ & 4 & 1 & 0 \\
\hline Delta TB (+) & 4 & 2 & $7^{*}$ & 0 \\
\hline \multicolumn{5}{|l|}{ Entacavir $(\mathrm{n}=70)$} \\
\hline Delta TB (-) & 41 & 2 & 11 & 3 \\
\hline Delta TB $(+)$ & 3 & 5 & 2 & 3 \\
\hline
\end{tabular}

${ }^{*} p<0.01$, significantly different between delta ALT (-) and delta ALT (+) in each antiviral therapy using Fisher's exact test.

(Fig. 1C, left). In G\#3 and G\#4, patients with abnormal TBmax showed two populations: abnormal TBmax and ALT amelioration versus abnormal TBmax and ALT exacerbation (Fig. 1C, right).

ALTmax and TBmax were compared in those who underwent adefovir and lamivudine therapy. In 83 adefovirtreated patients showing ALT greater than three times the ULN, 53 patients were prescribed with adefovir alone $(67.9 \%)$, whereas 30 patients were treated with adefovir following lamivudine (36.1\%). Of the patients with ALT more than three times the ULN but less than 15 times the ULN, TBmax (+) ALT (-) patients were 7 and 11, respectively, in the adefovir alone and adefovir after lamivudine therapy (4/46, 9\% vs. 4/22, 18\%), suggesting that adefovir after lamivudine treatment causes hyperbilirubinemia. Similar changes were observed in those showing ALT greater than 15 times the ULN (3/7, 43\% versus 7/8, 88\%) (Table 2). Overall, our results support the idea that lamivudine treatment may aggravate hyperbilirubinemia in a population undergoing severely advanced hepatitis.

Analysis of co-medications. To find any confounding factors potentially associated with lamivudine-induced bilirubinemia, the effects of concurrent medications, particularly those frequently prescribed with lamivudine, on ALTmax or TBmax were analyzed. Briefly, drugs co-prescribed with lamivudine were considered if prescribed within one month prior to measurement of ALTmax or TBmax. The average number of co-mediations was 4.3 drugs per day (drugs used at a frequency of $1 \%$ or less were excluded). In this analysis, silymarin, ursodeoxycholic acid (UDCA), Bease, entecavir, adefovir, prednisone, corticosteroid, tacrolimus, interferon, and/or zidovudine were often used with lamivudine, consistent with a case report (16). It has been reported that entecavir, adefovir, tacrolimus, interferon, and zidovudine treatments resulted in $110 \%$ increased risk of hepatotoxicity despite their low prescription frequencies (i.e., $<10 \%$ ). In $\mathrm{G} \# 3$ and $\mathrm{G} \# 4$, the proportion of co-medication of lamivudine with either silymarin or UDCA was $46 \%$. Their TBmax values were in an abnormal range, although ALT was improved. In G\#4, lamivudine plus UDCA treatment showed higher ALT and TBmax than did lamivudine treatment alone.

\section{DISCUSSION}

Adverse drug reactions have become an important clinical issue and a concern in the public health system, being responsible for $6.5 \%$ of all hospital admissions, with approximately one-fourth of these patients at risk for death $(17,18)$. The occurrence of ADRs has increased every year (4). As an effort to minimize ADRs, it is necessary to understand the causal relationship between medications and ADR incidence (2). Data acquisition from laboratory signals using patient EMRs may be of value to find ADRs and other conditions in patients (4). Three major methods are often used for ADR reports and include 1) spontaneous reports by clinicians or patients, 2) retrospective manual chart reviews, and 3) reviews of ADRs based on patient EMRs (5). Among them, reviews of EMR databases may be useful to assess the incidence of ADRs and to create an electronic alert system (7). A total of 4,690 reports of suspected druginduced liver injury associated with fatal outcomes was found using an EMR database in a hospital setting (19).

Lamivudine was the first registered antiviral drug and is widely used due to its efficacy during short-term administration (1). It is used as a mainstream therapy for $\mathrm{HBV}$ patients due to its effectiveness against HBV DNA and his- 
tological improvements. During anti-viral therapy, ALT is used as a biomarker to assess drug efficacy and is monitored as a general recovery factor. Despite its effectiveness, a few case reports have raised the possibility that lamivudine causes liver toxicity in certain populations $(20,21)$. In the current study, we report that patients with underlying severely advanced hepatitis suffered from hyperbilirubinemia despite ALT amelioration, presumably due to a limited capacity for bilirubin metabolism. Nonetheless, lamivudine medication did not exacerbate bilirubinemia in patients with no, mild, or moderate hepatitis. Our results here support the possibility that lamivudine undergoes biotransformation in the liver for clearance, particularly in those having severely advanced hepatitis. This idea is strengthened by the finding that lamivudine, which requires a larger dosing amount than other antiviral agents, may have the potential to cause druginduced liver injury (22). In this study, we paid attention to patients suffering from severely advanced hepatitis and diminished liver function, considering this possibility.

In many cases, abnormal increases of TB may result from overproduction of indirect bilirubin, inhibition of bilirubin uptake into hepatocytes, inhibition of bilirubin conjugation, and/or decreased excretion of direct bilirubin (23-26). In general, an abnormality is usually judged by TB content in the blood. In the present study, our findings raised the notion that lamivudine imposes a detoxifying burden (i.e., it inhibits bilirubin conjugation) to those with severe hepatitis, as indicated by exacerbation of hyperbilirubinemia despite ALT amelioration. This may be due to saturation of bilirubin biotransformation due to a decrease of hepatocytes and/or their dysfunction.

Our finding that lamivudine treatment had a deleterious effect and should be limited in its use for those with severe hepatitis is strengthened by our data showing improvements in both ALT and TB in populations having mild or moderate degrees of hepatitis. Unfortunately, the improvement in liver function when using lamivudine in patients with severe hepatitis was only $30 \%$ (3 out of 10 patients with chronic hepatitis $\mathrm{B} ; 1)$. This limitation may have been associated with deterioration of hepatocyte viability, possibly due to lamivudine-induced liver injury.

Antiviral agents including lamivudine and other drugs have nucleoside moieties (14), which may require oxidation and conjugation for detoxification. Studies using animals and cells showed that lamivudine metabolism depends on CYP2B6 and CYP3A and uridine diphosphate alpha-Dglucuronic acid conjugation (27), suggesting that competition between lamivudine (metabolite) and bilirubin for glucuronide conjugation in hepatocytes. In general, lamivudine biotransformation in the liver is a minor route of elimination in healthy individuals because unchanged parent drug molecules are cleared predominately by the kidney (8). However, patients with severely advanced hepatitis may have reduced functional liver function (28). Hence, hepatic metabolism of lamivudine may influence its glucuronidation in patients having limited and decreased liver function $(5 \sim 10 \%)$, presumably in conjunction with low plasma protein binding (due to low plasma albumin) (8). This may result from the first-order rate of uridine 5'-diphospho-glucuronosyltransferase conjugation (12). This contention matches with the finding that patients with severely advanced hepatitis have $35 \sim 48 \%$ viable hepatocyte mass (12). Moreover, hyperbilirubinemic severity and related jaundice may be assessed based on ALT and bilirubin levels in the blood. Excess degradation of heme and/or reduction of bilirubin excretion can also induce hyperbilirubinemia. Thus, hyperbilirubinemia may result from hemolysis, such as hemolytic anemia that increases bilirubin production, and/or from hepatic dysfunctions, such as abnormal liver function and impaired bilirubin metabolism and excretion (29). Thus, lamivudine may compete with bilirubin for organic anion transporter-mediated uptake into hepatocytes $(13,16)$, suggesting the possibility that a high concentration of lamivudine in the blood aggravates hyperbilirubinemia. Collectively, lamivudine-induced TB elevation in patients underlying severely advanced hepatitis may reflect metabolic burden, implying that caution should be exercised for lamivudine therapy in patients experiencing severely advanced hepatitis (i.e., ALT $>600 \mathrm{IU} / \mathrm{L}$ ). From our results, lamivudineinduced hyperbilirubinemia appeared to be irreversible in patients experiencing severely advanced hepatitis.

Of course, this conclusion has several limitations. Excluded factors, such as comorbidity, polypharmacy, gender, and age can affect the occurrence and severity of lamivudine-induced hyperbilirubinemia. In addition, patient information was collected from only one hospital and one specific period. These exclusions were indispensable to deduce the conclusions based on the EMR database used but it still remains a limitation of this conclusion.

In our study, adefovir and entecavir had no significant effect on TB (Table 2), presumably because they are mainly eliminated via the kidney $(16,28,30)$. Even in patients with advanced severe hepatitis, their metabolic burden for elimination seemed to be not great. Because of the resistance of lamivudine-only therapy and the cost-effectiveness of antiviral agents, combination therapy using lamivudine and adefovir is often recommended (30-34). In the present study, patients subjected to adefovir therapy after lamivudine indeed exhibited higher TB than those with adefovir alone (Table 2), supporting the identified lamivudine effect on hyperbilirubinemia. Overall, our results support the contention that a portion of patients experiencing severely advanced hepatitis need to be carefully monitored to prevent lamivudine-induced hyperbilirubinemia.

\section{ACKNOWLEDGMENTS}

This work was supported by the Education and Research 
Encouragement Fund of Seoul National University Hospital.

\section{CONFLICT OF INTEREST}

None to declare.

Received September 12, 2017; Revised September 21, 2017; Accepted September 25, 2017

\section{REFERENCES}

1. Uglietti, A., Zanaboni, D., Gnarini, M. and Maserati, R. (2013) Emtricitabine/tenofovir in the treatment of HIV infection: current PK/PD evaluation. Expert Opin. Drug Metab. Toxicol., 8, 1305-1314.

2. Andreea, F. and Marius, B. (2009) Adverse drug reactions in clinical practice: a causality assessment of a case of druginduced pancreatitis. J. Gastrointest. Liver Dis., 18, 353-358.

3. Jha, A.K., Kuperman, G.J., Teich, J.M., Leape, L., Shea, B., Rittenberg, E., Burdick, E., Seger, D.L., Vander Vliet, M. and Bates, D.W. (1998) Identifying adverse drug events: development of a computer-based monitor and comparison with chart review and stimulated voluntary report. J. Am. Med. Inform. Assoc., 5, 305-314.

4. Kohn, L.T., Corrgan, J.M. and Donaldson, M.S. (1999) To Err in Human: Building a Safer Health System, National Academy Press, Washington, D.C.

5. Lee, Y.H., Kang, U.G. and Park, R.W. (2008) Development of adverse drug event surveillance system using BI technology. Int. J. Contents, 9, 106-113.

6. Rodriguez-Monguio, R., Otero, M.J. and Rovira, J. (2003) Assessing the economic impact of adverse drug effects. Pharmacoeconomics, 21, 623-650.

7. Tisdale, J.E. and Miller, D.A. (2005) Drug-Induced Diseases. Prevention, Detection and Management, Ameritan Society of Health-System Pharmacist Press, Bethesda, Maryland, p. 1004.

8. Verhaz, A. (2014) Experience with lamivudine treatment for severe acute hepatitis B. Srp. Arh. Celok. Lek., 142, 703-707.

9. Ah, Y.M., Kim, Y.H., Kim, M.-J., Choi, Y.H., Park, K.H., Son, I.J. and Kim, S.G. (2008) Drug-induced hyperbilirubinemia and the clinical influencing factors. Drug Metab. Rev., 40, 511-537.

10. Kaplowitz, N. (2003) Drug-induced liver disorders: introduction and overview in Drug-Induced Liver Disease (Kaplowitz, N. and Deleve, L.D. Eds.). Marcel Dekker Inc., New York, pp. 150-158.

11. Zimmerman, H.J. (1999) Hepatotoxicity. The Adverse Effects of Drugs and Other Chemicals on the Liver (2nd edition), Lippincott Williams \& Wilkins, Philadelphia, pp. 235-249.

12. Pradeep Kumar, S., Medhi, S., Asim, M., Das, B.C., Gondal, R. and Kar, P. (2011) Evaluation of adefovir \& lamivudine in chronic hepatitis B: correlation with HBV viral kinetic, hepatic-necro inflammation \& fibrosis. Indian J. Med. Res., 133, 50-56.

13. Kudo, M., Todo, A., Ikekubo, K., Hino, M., Yonekura, Y., Yamamoto, K. and Torizuka, K. (2014) Functional hepatic imaging with receptor-binding radiopharmaceutical: clinical potential as a measure of functioning hepatocyte mass. Gastroenterol. Jpn., 26, 734-741.

14. Rowland, A., Mackenzie, P.I. and Miners, J.O. (2015) Transporter-mediated uptake of UDP-glucuronic acid by human liver microsomes: assay conditions, kinetics, and inhibition. Drug Metab. Dispos., 43, 147-153.

15. Wan, Z., Wu, Y., Yi, J., You, S., Liu, H., Sun, Z., Zhu, B., Zang, H., Li, C., Liu, F., Li, D., Mao, Y. and Xin, S. (2015) Combining serum cystatin $\mathrm{C}$ with total bilirubin improves short-term mortality prediction in patients with HBV-related acute-on-chronic liver failure. PLOS ONE, 10, e0116968.

16. Tsubota, A., Arase, Y., Saitoh, S., Kobayashi, M., Suzuki, Y., Suzuki, F., Chayama, K., Murashima, N., Ikeda, K., Kobayashi, M. and Kumada, H. (2001) Lamivudine therapy for spontaneously occurring severe acute exacerbation in chronic hepatitis B virus infection: a preliminary study. Am. J. Gastroenterol., 96, 557-562.

17. Pirmohamed, M., James, S., Meakin, S., Green, C., Scott, A.K., Walley, T.J., Farrar, K., Park, B.K. and Breckenridge, A.M. (2004) Adverse drug reactions as cause of admission to hospital: prospective analysis of 18820 patients. $B M J, \mathbf{3 2 9}$, 15-19.

18. Lazarous, J., Pomeranz, B. and Corey, P.N. (1998) Incidence of adverse drug reactions in hospitalized patients: a meta-analysis of prospective studies. JAMA, 279, 1200-1205.

19. Mauben, M., Madigan, D., Gerritis, C.M. (2007) The role of data mining in pharmacovigilance. Expert Opin. Drug Saf., 14, 929-948.

20. Barcena, R., Del Campo, S., Moraleda, G., Casanovas, T., Prieto, M., Buti, M., Moreno, J.M., Cuervas, V., Fraga, E., De la Mata, M., Otero, A., Delgado, M., Loinaz, C., Barrios, C., Dieguez, M.L., Mas, A., Sousa, J.M., Herrero, J.I., Muñoz, R., Avilés, J.F., Gonzalez, A. and Rueda, M. (2005) Study on the efficacy and safety of adefovir dipivoxil treatment in postliver transplant patients with hepatitis B virus infection and lamivudine-resistant hepatitis B virus. Transplant. Proc., 37, 3960-3962.

21. Ben-Ari, Z., Mor, E. and Tur-Kaspa, R. (2003) Experience with lamivudine therapy for hepatitis B virus infection before and after liver transplantation, and review of the literature. $J$. Intern. Med., 253, 544-552.

22. Liu, C., Ye, J., Jia, H., Zhang, M., Han, H., Chen, F. and Chen, C. (2013) Entecavir and lamivudine therapy for severe acute chronic hepatitis B. Exp. Ther. Med., 5, 545-548.

23. Bonkovsky, H.L., Azar, R., Bird, S., Szabo, G. and Banner, B. (2002) Severe cholestatic hepatitis caused by thiazolidinediones: risks associated with substituting rosiglitazone for troglitazone. Dig. Dis. Sci., 47, 1632-1637.

24. Brickford, C.L. and Spencer, A.P. (2005) Biliary sludge and hyperbilirubinemia associated with ceftriaxone in an adult: case report and review of the literature. Pharmacotherapy, 25, 1389-1395.

25. Thompson, P.D., Clarkson, P. and Karas, R.H. (2003) Statinassociated myopathy. JAMA, 289, 1681-1690.

26. Willens, H.J. (2000) Clopidogrel-induced mixed hepatocellular and cholestatic liver injury. Am. J. Ther., 7, 317-318.

27. Cousins, D. and Baker, M. (2004) The work of the National Patient Safety Agency to improve medication safety. Br. J. Gen. Pract., 54, 331-333. 
28. Wang, G.L., Liu, Y., Qiu, P., Zhou, S.F., Xu, L.F., Wen, P., Wen, J.B. and Xiao, X.Z. (2016) Cost-effectiveness of Lamivudine, Telbivudine, Adefovir Dipivoxil and Entecavir on decompensated hepatitis B virus-related cirrhosis. Eur. Rev. Med. Pharmacol. Sci., 20, 866-872.

29. Korenblat, K.M. and Berk, P.D. (2005) Hyperbilirubinemia in the setting of antiviral therapy. Clin. Gastroenterol. Hepatol., 3, 303-310.

30. Zhang, C., Ke, W., Liu, L., Gao, Y., Yao, Z., Ye, X., Zhou, S. and Yang, Y. (2016) Cost-effectiveness comparison of lamivudine plus adefovir combination treatment and nucleos(t)ide analog monotherapies in Chinese chronic hepatitis B patients. Drug Des. Devel. Ther., 10, 897-910.

31. Leung, N.W.Y., Lai, C.-L., Chang, T.-T., Guan, R., Lee, C.-M., Ng, K.-Y., Lim, S.-G., Wu, P.C., Dent, J.C., Edmundson, S., Condreay, L.D. and Chien, R.N. (2001) Extended lamivudine treatment in patients with chronic hepatitis B enhances hepatitis $\mathrm{B}$ e antigen seroconversion rates: results after 3 years of therapy. Hepatology, 33, 1527-1532.

32. Li, X., Jie, Y., You, X., Shi, H., Zhang, M., Wu, Y., Lin, G., Li, X., Gao, Z. and Chong, Y. (2015) Optimized combination therapies with adefovir dipivoxil (ADV) and lamivudine, telbivudine, or entecavir may be effective for chronic hepatitis $\mathrm{B}$ patients with a suboptimal response to ADV monotherapy. Int. J. Clin. Exp. Med., 8, 21062-21070.

33. Perrillo, R., Schiff, E., Yoshida, E., Statler, A., Hirsch, K., Wright, T., Gutfreund, K., Lamy, P. and Murray, A. (2000) Adefovir dipivoxil for the treatment of lamivudine-resistant hepatitis B mutants. Hepatology, 32, 129-134.

34. Peters, M.G., Hann, H.W., Martin, P., Heathcote, E.J., Buggisch, P., Rubin, R., Bourliere, M., Kowdley, K., Trepo, C., Gray, D.F., Sullivan, M., Kleber, K., Ebrahimi, R., Xiong, S. and Brosgart, C.L. (2004) Adefovir dipivoxil alone or in combination with lamivudine in patients with lamivudine-resistant chronic hepatitis B. Gastroenterology, 126, 91-101. 